\title{
artigo
}

Nascimento, C.M.M.; Santana, R.B.C.; Oliveira, A.P.S.; Araújo, M.G.R.; Guerino, M.R.; Paiva, M.G.;

Análise espaço-temporal, cinemática e cinética da marcha em indivíduos pós-acidente vascular cerebral

\section{Análise espaço-temporal, cinemática e cinética da marcha em indivíduos pós-acidente vascular cerebral}

\author{
Spatio-temporal, kinematic and kinetics gait analysis in post-stroke individuals
}

Análisis espacial temporal, cinemático y cinético de la marcha en individuos de accidentes vasculares postragenales

\section{RESUMO}

Objetivo: Analisar as características espaço-temporais, cinemáticas e cinéticas da marcha em indivíduos pós-AVC. Métodos: A amostra foi composta por 16 indivíduos (10 do grupo Pós-AVC e 6 do grupo Controle). A análise das variáveis da marcha, a amplitude de movimento, o momento e a potência do tornozelo foram obtidas por meio do Qualysis Track Manager ${ }^{\circledR}$, com 10 câmeras, filmando um percurso de 5 metros. Resultados: 0 grupo Pós-AVC apresentou uma velocidade, aproximadamente, 50\% menor que o grupo Controle $(p<0,0001)$. Em relação aos cinéticos, o momento e a potência do tornozelo do lado não-parético foi maior que o parético, mas menor que o grupo Controle $(p<0,05)$. Entretanto, na cinemática não ocorreu diferença intergrupo. Na cinemática não ocorreu diferença intergrupo. Conclusão: As alterações da marcha no pós-AVC podem ter ocorrido por desuso da musculatura no lado parético, provavelmente desencadeando o fenômeno da plasticidade muscular, no qual ocorrem mudanças na expressão fenotípica de fibras musculares.

DESCRITORES: Análise de Marcha; Acidente Vascular Cerebral; Plasticidade Neuronal.

\section{ABSTRACT}

Objective: To analyze the spatio-temporal, kinematic and kinetic characteristics of gait in post-stroke individuals. The sample consisted of 16 individuals (10 from the Post-stroke group and 6 from the Control group). The analysis of gait variables, range of motion, moment and power of the ankle were obtained using Qualysis Track Manager ${ }^{\circledR}$, with 10 cameras, filming a 5-meter course. Results: The Post-stroke group showed a speed approximately 50\% lower than the Control group ( $<0.0001)$. With respect to kinetics, the non-paretic side of ankle moment and power were higher than paretic side, but lower than control group. ( $p<0.05$ ). In kinematics there was no intergroup difference. Conclusion: Changes in gait in the post-stroke period may have occurred due to muscle disuse on the paretic side, probably triggering the phenomenon of muscle plasticity, in which changes in the phenotypic expression of muscle fibers occur.

DESCRIPTORS: Gait Analysis; Stroke; Neuronal Plasticity.

\section{RESUMEN}

Objetivo: Analizar las características espacio-temporales, cinemáticas y cinéticas de la marcha en individuos post-ictus. Métodos: La muestra estuvo formada por 16 individuos (10 del grupo Post ictus y 6 del grupo Control). El análisis de las variables de la marcha, rango de movimiento, momento y potencia del tobillo se obtuvo mediante Qualysis Track Manager ${ }^{\circledR}$, con 10 cámaras, filmando un recorrido de 5 metros. Resultados: El grupo Post-ACV mostró una velocidad aproximadamente un 50\% menor que el grupo Control $(p<0,0001)$. En cuanto a la cinética, el momento y la potencia del tobillo en el lado no parético fue mayor que en el parético, pero menor que en el grupo Control $(p<0.05)$. Sin embargo, en cinemática no hubo diferencia entre grupos. En cinemática no hubo diferencia intergrupal. Conclusión: Los cambios en la marcha en el período posterior al ictus pueden haber ocurrido debido al desuso muscular en el lado parético, probablemente desencadenando el fenómeno de plasticidad muscular, en el cual ocurren cambios en la expresión fenotípica de las fibras musculares.

DESCRIPTORES: Análisis de la marcha; Accidente vascular cerebral; Plasticidad Neuronal.

RECEBIDO EM: 20/03/2021 APROVADO EM: 09/04/2021

\section{Camila Maria Mendes Nascimento}

Fisioterapeuta, Mestranda em Gerontologia pela Universidade Federal de Pernambuco, Recife, Pernambuco, Brasil.

ORCID: 0000-0002-2223-4196 


\section{Ricardo Vinícius de Carvalho Santana}

Fisioterapeuta, Residência Multiprofissional em Fisioterapia em Terapia Intensiva do Hospital Agamenon Magalhães, Recife, Pernambuco, Brasil.

ORCID: 0000-0003-4791-1154

\section{Ana Paula Silva de Oliveira}

Fisioterapeuta, Mestre em Engenharia Biomédica pela Universidade Federal de Pernambuco, Recife, Pernambuco, Brasil. ORCID: 0000-0003-3746-7504

\section{Maria das Graças Rodrigues de Araújo}

Fisioterapeuta, Professora Doutora do Departamento de Fisioterapia, Universidade Federal de Pernambuco, Recife, Pernambuco, Brasil. ORCID: 0000-0002-9980-6172

\section{Marcelo Renato Guerino}

Fisioterapeuta, Professor Doutor do Departamento de Fisioterapia, Universidade Federal de Pernambuco, Recife, Pernambuco, Brasil. ORCID: 0000-0002-3439-9166

\section{Maria das Graças Paiva}

Fisioterapeuta, Professora Doutora do Departamento de Fisioterapia, Universidade Federal de Pernambuco, Recife, Pernambuco, Brasil. ORCID: 0000-0001-6913-8639

\section{INTRODUÇÃO}

A marcha é uma das funções mais utilizadas pelas pessoas; ela possibilita o deslocamento, a realização de atividades diárias, a manutenção da independência e da qualidade de vida. Podem ocorrer alterações patológicos que contribuem para a diminuição da sua eficiência e aumento de gasto energético como o Acidente Vascular Cerebral (AVC), onde após a lesão ocorrem dificuldades no controle voluntário do movimento comprometendo força muscular, tônus, coordenação, equilíbrio e percepção ${ }^{1}$.

Cerca de $70 \%$ retomam a sua capacidade de deambular, embora sem o sinergismo muscular adequado, além de disfunções metabólicas musculares, ocorridas no lado parético, associadas ao desenvolvimento da espasticidade ocasionam modificações sensório-motoras, mudando assim a plasticidade da musculatura acometida ${ }^{2}$.

A plasticidade muscular esquelética é altamente maleável e exibe notável capacidade de ajuste quando o músculo sofre contração, estando suscetível a modificar suas características fenotípicas e proporcionar melhor adaptação funcional ${ }^{3}$. Dentre as alterações decorrentes dos estímulos aplicados sobre o músculo, temos a transição das fibras lentas para as rápidas no lado parético e maior estímulo para as fibras lentas no não-parético. Dessa forma, em bipedestação, o centro de gravidade desses indivíduos é desviado para este lado e, associado aos déficits de equilíbrio, propriocepção e controle seletivo, ocasiona diminuição na distribuição de peso no lado parético ${ }^{4}$. Disso decorre reduções na velocidade, na cadência e no comprimento da passada, além de aumentos relativos na duração do ciclo da marcha e nos períodos de duplo apoios.

Diversas ferramentas são utilizadas para investigar a biomecânica do movimento. A análise tridimensional desse evento é um recurso empregado como método de pesquisa e avaliação da marcha normal ou patológica, permitindo verificar as características espaço-temporais como comprimento, duração da passada e velocidade $^{6}$. A velocidade pode ser definida como a distância que o corpo percorre para frente na unidade de tempo; a duração da passada, como o momento desde o contato inicial de um membro até o contato inicial seguinte do mesmo membro; e o comprimento da passada é a distância percorrida de dois toques sucessivos do mesmo pé7.

O estudo da biomecânica da marcha no pós-AVC fornece uma base para a compreensão das possíveis alterações nos mecanismos do movimento e, a análise quantitativa dessa marcha, pode ser utilizada como um instrumento para diagnóstico cinético-funcional, bem como, para o planejamento fisioterapêutico, considerando a individualidade de cada caso. Assim, o objetivo deste estudo foi analisar, quantitativamente, as características cinemáticas e cinéticas da marcha em indivíduos pós-AVC, caracterizando e diferenciando os seus padróes espaço-temporais.

\section{MÉTODO}

Foi realizado um estudo transversal de caráter exploratório, desenvolvido no Laboratório de Agilidade Humana, no Departamento de Fisioterapia e Ciências do Movimento Humano da Northwestern University, Chicago - Illinois (EUA). Foram selecionados indivíduos do Rehabilitation Institute of Chicago (RIC), de ambos os sexos e entre 50 e 70 anos de idade para os dois grupos de maio a julho de 2015. Grupo Pós-AVC ( $n=10)$ : foram elegíveis indivíduos por um período maior ou igual a 12 meses de lesão, sem nenhuma intervenção cirúrgica nos últimos 24 meses desde o início da coleta de dados, com a capacidade de andar sem o uso de dispositivo auxiliar de marcha durante o experimento. Como critérios de exclusão, os indivíduos com alguma outra lesão neurológica, déficit cognitivo, ou lesão osteomioarticular no membro inferior. Grupo 
A análise estatística foi realizada pelo software BioEstat $v$.

5.3. Média e desvio padrão (DP) foram calculados para todos os parâmetros estudados.

Controle $(n=6)$ : foi composto por indivíduos saudáveis, sem nenhuma patologia neurológica ou traumato-ortopédica. Todos os participantes assinaram o Termo de Consentimento Livre e Esclarecido, os quais foram previamente aprovados pelo Institutional Review Board da Northwestern University (IRB \# STU00200422).

Os dados antropométricos avaliados foram altura e peso. A altura foi mensurada, em centímetros $(\mathrm{cm})$, pelo software Visual $3 \mathrm{D}^{\circ}$ v. 5.0 (C-Motion, Inc., Germantown, MD, USA), e o peso, verificado, em quilogramas $(\mathrm{Kg})$, por uma plataforma de força $\mathrm{AMTI}^{\circ}$ (Force and Motion, Inc., Watertown, MA, USA), de precisão, associada ao software Qualysis Track Manager ${ }^{\circ}$ - (QTM) v. 2.1 (Motion Capture Systems, Inc., Gothenburg, SWEDEN). A análise de marcha foi realizada através do QTM para a obtenção dos seus parâmetros espaço-temporais, cinemáticos e cinéticos. Este programa é um sistema de análise de fotogrametria baseado em vídeo, composto por 10 câmeras optoeletrônicas. Ele permite a reconstrução tridimensional da imagem com marcadores refletores esféricos localizados em 46 pontos no corpo, para a análise em posição ortostática, de acordo com um modelo esquelético criado no Visual 3D'.
Antes de iniciar o experimento os indivíduos foram instruídos a andarem em uma velocidade confortável, percorrendo 5 metros de comprimento. Durante o percurso havia seis plataformas de força $\mathrm{AMTI}^{\circ}$ para captação das variáveis cinéticas. Todos realizaram 20 tentativas, sendo a primeira considerada como familiarização do ambiente experimental. Os indivíduos vestiram um colete ajustável, ao qual era acoplado um dispositivo de suporte Zero G Lite v. 2.0 (Aretech LLC, Ashburn, VA, USA), de forma a prevenir quedas ou desequilíbrio, mas não haveria suporte de peso.

Todos os dados foram calculados no Visual $3 \mathrm{D}^{\circ}$ e Matlab $^{\circ}$ v. 8.5 (The Mathworks, Natick, MA, USA). No Visual $3 \mathrm{D}$, os parâmetros espaço-temporais passaram por um filtro passa-baixa Butterworth, com uma frequência de corte de $6 \mathrm{~Hz}$ além de serem normalizados numa escala de 0 a $100 \%$ do ciclo de marcha. No Matlab foram obtidos os dados de média dos parâmetros cinemáticos e cinéticos. Os dados espaço-temporais calculados foram: velocidade de marcha, largura da passada, tempo da fase de apoio, tempo da fase de balanço e tempo do duplo apoio. Já o cinemático e os cinéticos foram: ângulo, momento e potência do tornozelo, respectivamente, e foram calculados durante a fase de apoio de cada membro inferior de cada grupo. Apenas os dados no plano sagital foram analisados para identificar as alterações mais frequentes dos indivíduos pós-AVC.

Tabela 1. Características gerais da amostra estratificada de acordo com os grupos Pós-AVC e Controle.

\section{VARIÁVEIS}

Gênero (M/F)

Idade (Anos)

Massa (kg)

IMC (kg/m2)

Lado Parético (D/E)

Tempo de Lesão (meses) corporal; $\mathrm{D}=$ Direito; $E=$ Esquerdo
A análise estatística foi realizada pelo software BioEstat v. 5.3. Média e desvio padrão (DP) foram calculados para todos os parâmetros estudados. O teste de normalidade de Kolmogorov-Smirnov foi realizado previamente à análise estatística para confirmar a distribuição normal dos dados. Apenas a variável tempo da fase de apoio, quando comparados os lados parético e não-parético do grupo Pós-AVC, apontou distribuição não-paramétrica. Então, para este parâmetro foi realizado o teste de Wilcoxon. Quanto aos demais parâmetros, posteriormente, foram executados o teste-T Student (teste paramétrico) para amostras relacionadas nas análises intragrupo, e o teste-T Student para amostras independentes nas análises intergrupo. Foi realizada análise estatística, relacionando o membro inferior direito com o esquerdo do grupo Controle. Por não haver diferença significativa, as comparações intergrupo foram realizadas com a média dos membros do grupo Controle. O nível de significância foi mantido com um valor de $\mathrm{p}<0,05$.

\section{RESULTADOS}

A média de idade entre os participantes foi de 60,10 $\pm 4,20$ anos entre o grupo

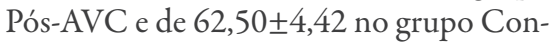
trole. Houve uma distribuição homogênea quanto ao sexo, à altura e à massa corporal em ambos os grupos os registros foram semelhantes, conforme descrito na tabela 1 . 
Todos os parâmetros espaço-temporais mostraram diferença estatisticamente significativa $(p<0,05)$, quando analisados entre os grupos. Todos os indivíduos do grupo Pós-AVC apresentaram velocidade, aproximadamente, 50\% menor que os indivíduos do grupo controle $(p<0,0001)$. Em contrapartida, o grupo Pós-AVC passou cerca de duas vezes mais tempo com os pés no chão ( $\mathrm{p}=0,0069)$ no início do ciclo da marcha (tempo de duplo apoio), além de maior largura da passada $(\mathrm{p}=0,001)$, em relação ao grupo Controle, visto na tabela 2.

Em geral, os lados parético e não-parético do grupo Pós-AVC exibiram diferença significativa nos outros parâmetros verificados, como os tempos das fases de apoio e de balanço. De fato, o lado parético teve um tempo de apoio menor que o lado não-parético $(\mathrm{p}=0,0051)$, corroborado por um tempo de balanço maior ( $\mathrm{p}=0,0011$ ). Quando comparados com o Controle, apresentaram tempo de apoio maior e de balanço maior, tanto no lado parético ( $\mathrm{p}=0,0259 ; \mathrm{p}=0,0052)$ como no não-parético ( $\mathrm{p}=0,0115 ; \mathrm{p}=0,0047)$, como demonstra a tabela 3 .

A média da amplitude de movimento (ADM) do tornozelo foi homogênea quando comparados os lados parético ( $\mathrm{p}=0,0967)$ e não-parético $(\mathrm{p}=0,4507)$ e o grupo Controle, não sendo observada diferença significativa. Entretanto, quando comparados os lados parético e não-parético, as médias registradas foram 73,00 $(7,64)$ e $82,43(5,67)$, respectivamente. A ADM do tornozelo, no lado parético, foi consideravelmente menor $(\mathrm{p}=0,0075)$ que no lado não-parético.

Em relação ao momento e potência do tornozelo, as médias tanto intra- como intergrupo apresentaram diferença significativa. O momento do tornozelo no lado parético foi expressivamente menor ( $\mathrm{p}=0,0031)$ que no lado não-parético. Todavia, o grupo Controle revelou em

Tabela 2. Parâmetros espaço-temporais dos grupos Pós-AVC e Controle

$\begin{array}{llcc}\text { PARÂMETROS ESPAÇO- } & & \text { GRUPO PÓS-AVC } & \text { GRUPO CONTROLE } \\ \text { TEMPORAIS } & & & \\ \text { Velocidade de Marcha (m/s) } & \text { Média (DP) } & 0,58(0,16)^{*} & 1,25(0,22)^{*} \\ \text { Tempo do Duplo Apoio (s) } & \text { Média (DP) } & 0,54(0,28)^{*} & 0,23(0,07)^{*} \\ \text { Largura da Passada (m) } & \text { Média (DP) } & 0,20(0,05)^{*} & 0,13(0,01)^{*} \\ \text { Fonte: a pesquisadora (2016); DP: Desvio Padrão *diferença significativa (p<0,05) } & \end{array}$

Tabela 3. Parâmetros espaço-temporais intra- e intergrupo.

$\begin{array}{lllll}\text { PARÂMETROS ESPAÇO- } & & \text { LADO } & \text { LADO NÃO- } & \text { GRUPO } \\ \text { TEMPORAIS } & & \text { PARÉTICO } & \text { PARÉTICO } & \text { CONTROLE } \\ \text { Tempo da Fase de Apoio (s) } & \text { Média (DP) } & 0,91(0,29)^{*} & 1,10(0,38)^{*} & 0,66(0,09)^{*} \\ \text { Tempo da Fase de Balanço (s) } & \text { Média (DP) } & 0,57(0,13)^{*} & 0,37(0,06)^{*} & 0,43(0,04)^{*} \\ \text { Largura da Passada (m) } & \text { Média (DP) } & 0,20(0,05)^{*} & 0,13(0,01)^{*} & \\ \text { Fonte: a pesquisadora (2016); DP: Desvio Padrão *diferença significativa (p<0,05) } & & \end{array}$

Tabela 4. Parâmetros cinemático e cinéticos do tornozelo dos grupos Pós-AVC e Controle.

\begin{tabular}{|c|c|c|c|c|}
\hline PARÂMETRO CINEMÁTICO & & $\begin{array}{l}\text { LADO } \\
\text { PARÉTICO }\end{array}$ & $\begin{array}{l}\text { LADO NÃO- } \\
\text { PARÉTICO }\end{array}$ & $\begin{array}{l}\text { GRUPO } \\
\text { CONTROLE }\end{array}$ \\
\hline ADM do Tornozelo $\left({ }^{\circ}\right)$ & Média (DP) & $73,00(7,64)^{*}$ & $82,43(5,67)$ & $79,90(7,28)$ \\
\hline \multicolumn{5}{|l|}{ Parâmetros Cinéticos } \\
\hline Momento do Tornozelo (Nm/Kg) & Média (DP) & $0,46(0,16)^{*}$ & $0,64(0,16)^{*}$ & $0,81(0,11)^{*}$ \\
\hline Potência do Tornozelo (W/Kg) & Média (DP) & $0,27(0,16)^{*}$ & $0,93(0,39)^{*}$ & $1,47(0,30)^{*}$ \\
\hline
\end{tabular}

torno do dobro do momento do lado parético $(\mathrm{p}=0,0005)$, sendo também maior que no lado não-parético $(\mathrm{p}=0,0485)$. No que se refere a potência articular, notamos diferença sensível em ambos os membros dos indivíduos pós-AVC ( $\mathrm{p}<0,0001)$, assim como, o lado não-parético foi menor $(\mathrm{p}=0,0108)$ que no grupo Controle, mas também cinco vezes maior no grupo Controle sobre o lado parético $(\mathrm{p}<0,0001)$, de acordo com a tabela 4.

\section{DISCUSSÃO}

A velocidade de marcha é frequentemente utilizada para caracterizar os indivíduos pós-AVC, sendo o parâmetro de referência para avaliação e tratamento. Em detrimento disso, Lauzière et al. (2014) afirmaram que $60 \%$ dos indivíduos pós-AVC apresentam assimetria espaço-temporal, sendo geralmente investigados os tempos das fases de apoio e de balanço e de duplo apoio, como foi evidenciado em nosso estudo 8 .

De fato, verificamos menor velocidade dos indivíduos pós-AVC, ocasionando menor tempo da fase balanço no grupo Controle e no lado não-parético do grupo Pós-AVC e, consequentemente, maior duração da fase de apoio no grupo Pós-AVC em relação ao Controle, sendo algumas das estratégias tomadas para manutenção da estabilidade corporal. Na pesquisa de Lopes et al. (2015) foi observado que o lado não-parético de 21 indivíduos pós-AVC passou menor tempo de balanço que o lado parético, atribuindo isso a uma diferença na força muscular e percepção do lado parético, alterando a simetria da marcha9 .

Por outro lado, Dyer et al. (2014) afirmaram ocorrer menor tempo de apoio no lado parético em relação ao lado não-parético e também ao grupo Controle ${ }^{10}$. Evidências sugerem que tanto o exercício como a idade são fatores que contribuem para variação na proporção de fibras musculares, em que as do tipo II passam a ter propriedades tipo I, o que modifica seu fenótipo. No entanto, após um AVC as fibras musculares do lado parético estão mais propensas a alterarem suas caracte- 
rísticas do tipo I para o tipo II, fenômeno esse denominado plasticidade muscular ${ }^{11}$.

Sheffler et al. (2014) sugeriram que esses achados são influenciados não apenas pelo nível de lesão, mas sobretudo por uma velocidade reduzida. Em seu estudo, corroborando os nossos desfechos, eles verificaram diminuição da velocidade e cadência, maior tempo de duplo apoio e menor comprimento da passada em 108 indivíduos, não havendo associação desses resultados com um aumento do IMC dos envolvidos, relacionando os efeitos negativos desse aumento a um maior tempo ou distância de caminhada ${ }^{12}$. No entanto, outras pesquisas afirmaram não haver diferença significativa no tempo do duplo apoio de indivíduos pós-AVC sobre indivíduos saudáveis ${ }^{13}$.

Observamos em nossos achados que o grupo Pós-AVC apresentou maior largura da passada que o Controle. Tal fato pode ser devido as estratégias motoras escolhidas para se adaptar as demandas da tarefa. Por não conseguir realizar a transferência de peso adequada, há diminuição da velocidade e aumento do tempo onde o peso é transferido para o lado parético, resultando em uma passada mais larga. Alguns autores chamam à atenção sobre o tempo de apoio mais curto e passada mais larga do lado parético, e estas compensações produzem um deslocamento anormal do centro de gravidade, acarretando maior gasto energético ${ }^{14}$.

Além dos parâmetros espaço-temporais, alguns estudos relatam as alterações cinemáticas. Em nosso estudo nos detivemos à articulação do tornozelo, em que sua amplitude de movimento no lado parético foi menor que no lado não-parético, demonstrando perda na qualidade da simetria do movimento dessa articulação. Milovanovic (2012) explica que esses fatos são consistentes com o padrão anormal característico das articulações desses indivíduos, em virtude da elevação da pelve e circundação do quadril contralateral durante a fase de balanço ${ }^{14}$.

Contudo, nenhuma diferença cinemática foi encontrada entre os grupos da nossa pesquisa. Burdett et al (1988) encontraram, em 19 indivíduos com e sem órtese, maior
Observamos em nossos achados que o grupo Pós-AVC apresentou maior

largura da passada que o Controle.

\section{Tal fato pode ser}

devido as estratégias

motoras escolhidas

para se adaptar

as demandas da

tarefa. Por não

conseguir realizar

a transferência de

peso adequada,

há diminuição da

velocidade e aumento

do tempo onde $o$

peso é transferido

para o lado parético,

resultando em uma

passada mais larga. flexão plantar no contato inicial e balanço médio, e menor na retirada dos dedos ${ }^{15}$.

As alteraçôes cinéticas também foram observadas como mecanismos de compensação para estabilidade dos indivíduos. Em nossa análise percebemos um menor momento articular no lado parético, tanto intra- como intergrupo. Isso pode ser justificado pelo fato de que em média $70 \%$ dos indivíduos pós-AVC mostram alteração biomecânica no tornozelo, impedindo seu posicionamento adequado e dificultado a dorsiflexão do pé durante a fase de apoio ${ }^{16}$.

Kim (2003) selecionou 20 indivíduos pós-AVC e identificou que o lado parético apresentou momentos do quadril, joelho e tornozelo menores que o não-parético. A média dos momentos no lado parético variou de $21 \%$ a $83 \%$ em relação ao não-parético, com a maior assimetria encontrada no tornozelo ${ }^{17}$. Ele sugeriu que o momento de flexão plantar é a variável mais importante para predizer e explicar a variação da velocidade de $67 \%$ a $72 \%$. Entretanto, 7 dos participantes necessitaram de dispositivo auxiliar, o que pode ter alterado os resultados expostos ${ }^{18}$.

Outros pesquisadores avaliaram a potência do tornozelo de 12 indivíduos pós-AVC e 10 indivíduos saudáveis, subdivididos em dois grupos, de acordo com sua velocidade. Eles explicaram que, no lado parético, ocorre maior geração de potência no quadril para suprir a fraqueza muscular dos flexores plantares, por desuso e alteração de suas fibras musculares lentas para rápidas ${ }^{19}$.

Entre as dificuldades e limitações encontradas neste estudo, uma se refere ao tamanho da amostra, que ao se apresentar em número reduzido, pode ter impossibilitado resultados mais precisos e consistentes com os relatos na literatura. Além disso, observou-se que embora o estudo da locomoção dos indivíduos pós-AVC tenha motivado a realização de muitos estudos a maioria deles desconsidera a variabilidade que pode ocorrer entre a marcha desses indivíduos, apesar da aparência visual demonstrar similaridades.

Apesar disso, o diferencial em nossa pesquisa refere-se ao fato de que a propos- 
ta não se restringiu a comparar as possíveis diferenças nos parâmetros de marcha entre os grupos pós-AVC e Controle. Por certo, foi levado em consideração, as alterações ocorridas de um lado, dito "afetado", dos indivíduos pós-AVC, com o dito "não-afetado", e deste com o Controle. Com isso, observamos as variações funcionais na marcha em nível não apenas intergrupo, como também intragrupo, vislumbrando novas perspectivas de abordagem fisioterapêutica para esse tipo de paciente.

Mesmo assim, sugere-se que estudos de maior complexidade, utilizando ferramentas como a Eletromiografia, possam trazer respostas mais precisas a respeito do que ocorre nos membros inferiores desses indivíduos, em nível muscular, para elucidar as causas das alterações observadas

\section{Por certo, foi levado} em consideração, as alterações ocorridas de um lado, dito "afetado", dos indivíduos pós-AVC, com o dito "nãoafetado", e deste com o Controle. e explicitar a relação com o fenômeno da plasticidade muscular. Tais conhecimentos poderão contribuir para que os profissionais possam diferenciar os déficits primários das compensações oriundas dos ajustes que surgirão ao longo do tempo da realização da marcha no pós-AVC.

\section{CONCLUSÃO}

O método de análise de marcha utilizado neste estudo foi capaz de verificar as alterações observadas nos parâmetros avaliados, tanto intra- como intergrupo. Dessa maneira, compreender essas modificações pode contribuir para o acompanhamento da evolução e tratamento dos indivíduos após um AVC, assim como, traçar melhores estratégias terapêuticas em cada situação.

\section{REFERÊNCIAS}

1.Westpha PJ, Ferreira J, Schimit VM, Cecheti F, Bonetti LV, Saccani R. Análise cinemática da marcha em indivíduos com hemiparesia espástica após acidente vascular cerebral. Sci Med. 2016;26(2):1-7.

2.Hood DA, Irrcher I, Ljubicic V, Joseph AM. Coordination of metabolic plasticity in skeletal muscle. J Exp Biol. 2006;209(12):2265-75.

3.Boff SR. A fibra muscular e fatores que interferem no seu fenótipo. Acta fisiátrica. 2008; 15(2):111-116.

4.Styer-Acevedo J. Fisioterapia para crianças com paralisia cerebral. In: Tecklin JS, editor. Fisioterapia pediátrica. 3a ed. Porto Alegre: Artmed; 2002. p. 98-140.

5.Araújo AGN, Andrade LM, Barros RML. Sistema para análise cinemática da marcha humana baseado em videogrametria. Fisioter Pes. 2005;11(1):3-10.

6.Moura EWD, Lima E, Borges D, Silva PDAC. Fisioterapia: aspectos clínicos e práticos da reabilitação. In Fisioterapia: aspectos clínicos e práticos da reabilitação. Artes Médicas. 2010, p.615-42.

7.Shumway-Cook A, Woollacott MT. Controle Motor. Teoria e Aplicações. São Paulo: Manole, 2003, p. 289-380.

8.Lauziere S, Betschart M, Aissaoui R, Nadeau S. Understanding spatial and temporal gait asymmetries in individuals post stroke. Int J of Phys Med \& Rehabili, 2014: 1-11.

9.Lopes PG, Lopes JA, Brito CM, Alfieri FM, Rizzo Battistella L. Relationships of Balance, Gait Performance, and Functional Outcome in Chronic Stroke Patients: A Comparison of Left and Right Lesions. Biomed Res Int. 2015: 1-8.

10.Dyer JO, Maupas E, de Andrade Melo S, Bourbonnais D, Nadeau $S$, Forget R. Changes in activation timing of knee and ankle extensors during gait are related to changes in heteronymous spinal pathways after stroke. J Neuroeng Rehabil. 2014;11(148): 1-17.

11.Scherbakov N, Von Haehling S, Anker SD, Dirnagl U, Doehner W. Stroke induced Sarcopenia: muscle wasting and disability after stroke. International journal of cardiology. 2013;170(2), 89-94.

12.Sheffler LR, Bailey SN, Gunzler D, Chae J. Effect of body mass index on hemiparetic gait. PMC. 2014;6(10):908-13.

13.Lucarelli PRG, Greve JMA. An Alteration of the load-response mechanism of the knee joint during hemiparetic gait following stroke analyzed by 3-dimensional kinematic. Clinics 2006;61(4):295-300.

14. Milovanović I, Popović DB. Principal component analysis of gait kinematics data in acute and chronic stroke patients. Comput Math Methods Med. 2012;1-8.

15.Burdett RG, Borello-France D, Blatchly C, Potter C. Gait comparison of subjects with hemiplegia walking unbraced, with ankle-foot orthosis, and with Air-Stirrup brace. Phys Ther. 1988;68(8):1197-203.

16.Oken O, Yavuzer G. Spatio-temporal and kinematic asymmetry ratio in subgroups of patients with stroke. Eur J Phys Rehabil Med. 2008;44(2):127-32.

17.Kim CM, Eng JJ. The relationship of lower-extremity muscle torque to locomotor performance in people with stroke. Phys Ther. 2003;83(1):49-57.

18. Jonkers I, Delp S, Patten C. Capacity to increase walking speed is limited by impaired hip and ankle power generation in lower functioning persons post-stroke. Gait Posture. 2009;29(1):129-37.

19. Boudarham J, Roche N, Pradon D, Bonnyaud C, Bensmail D, Zory R. Variations in kinematics during clinical gait analysis in stroke patients. PLoS One. 2013;8(6):1-9. 